\title{
On the rise of small air bubbles in water
}

\author{
By P. G. SAFFMAN \\ Trinity College, Cambridge \\ (Received 9 February 1956)
}

\begin{abstract}
SUMmaRY
This paper is concerned with the motion in water of air bubbles whose equivalent spherical radii are in the range $0.5-4.0 \mathrm{~mm}$. These bubbles are not spherical but are, approximately, oblate spheroids; and they may rise steadily in a vertical straight line, or along a zig-zag path, or in a uniform spiral. The rectilinear motion occurs when the radius is less than about $0.7 \mathrm{~mm}$, and the other motions occur for larger bubbles. There is disagreement in the literature as to whether it is the zig-zag or the spiral motion that occurs. It was found experimentally that, when the bubbles are produced in the manner described in this paper, only the zig-zag motion occurs when the radius of the bubble is less than about $1 \mathrm{~mm}$, but bubbles of larger radius either zig-zag or spiral depending upon various factors.

The spiralling bubble is treated theoretically by assuming that the flow near the front of the bubble is inviscid (the Reynolds number of the motion is several hundred) and considering the distribution of pressure over the front surface. Equations are obtained relating the geometrical parameters of the spiral, the shape of the bubble and the velocity of rise. The analysis is simplified by assuming that the pitch of the spiral is large compared with its radius, and the velocity of rise and shape of the bubble are determined as functions of the radius. The experimental and theoretical values are compared, and fair agreement found. Reasons to account for the disagreement are proposed.

A modification of the theory is proposed to take account of the presence of impurities or surface-active substances in the water, and the velocities of rise thus predicted are in agreement with the experimental observations.

The zig-zag motion is treated in a similar way, and the analysis leads to an equation which determines the stability of the rectilinear motion. The value of the Weber number at which the rectilinear motion becomes unstable is deduced, and is found to be in fair agreement with experiment. The experimental evidence on the wake behind solid bodies is described briefly, and reasons are given for suggesting that the zig-zag motion is due to an interaction between the instability of the rectilinear motion and a periodic oscillation of the wake.
\end{abstract}




\section{INTRODUCTION}

Many authors have studied experimentally the motion of air bubbles in water and other liquids. Datta, Napier \& Newitt (1950) and Peebles \& Garber (1953) describe briefly many of these experiments, and further experiments have been carried out by Haberman \& Morton (1953). It has been observed that when the bubble radius is about $0.7 \mathrm{~mm}$ and the Reynolds number is several hundred, the character of the motion changes. Bubbles of radius less than $0.7 \mathrm{~mm}$ rise steadily in a straight line; but, when the radius exceeds $0.7 \mathrm{~mm}$, the bubble either zig-zags from side to side or rises in a spiral which appears to be quite uniform. (It is convenient to specify the size of an air bubble by its equivalent spherical radius, this being the radius of a sphere with the same volume as the bubble. Whenever the radius of a bubble is mentioned in this paper, the equivalent spherical radius is implied.) The various experimenters are not in agreement as to whether the bubbles spiral or zig-zag, and an experiment was performed (described below in $\$ 2$ ) in order to investigate this matter further.

The reported experimental evidence indicates that the frequency of the zig-zag motion is about seven per second and independent of the size of the bubble; the angular velocity of the spiralling motion also appears to be independent of the size and is about 30 radians per second, which corresponds to about five revolutions per second about the axis of the spiral. The radius of the spiral appears to decrease slowly as the bubble size increases, being about $1.5 \mathrm{~mm}$ when the radius of the bubble is about $1.0 \mathrm{~mm}$; the amplitude of the zig-zag is also of this order of magnitude. As the radius of the bubble increases past $2.0 \mathrm{~mm}$, the spiral or zig-zag begins slowly to disappear; and by the time the bubble radius is about $3.0 \mathrm{~mm}$, the spiral or zig-zag motion has disappeared completely. The motion is then roughly rectilinear, and continues to be so as the bubble radius increases, although the shape of the bubble alters considerably.

Small bubbles of radius less than $0.5 \mathrm{~mm}$ appear to be spherical. Larger bubbles are distorted, and the distortion becomes greater as the size increases further. Bubbles of radius between $0.5 \mathrm{~mm}$ and $3.0 \mathrm{~mm}$ are oblate spheroids approximately, but the shape of the larger bubbles in this size range tends to fluctuate somewhat. For a bubble of radius larger than $3.0 \mathrm{~mm}$, the shape is very irregular and fluctuates considerably; then, at a radius of about one centimetre, the bubble has the shape of a spherical cap, and this shape is maintained as the bubble radius increases.

The velocities of rise have been measured for spiralling and zig-zagging bubbles. The measurements show a fair amount of scatter, but there appears to be no systematic difference between the velocities of spiralling or zig-zagging bubbles, i.e. the velocity appears to be independent of the nature of the motion. Several authors (e.g. Haberman \& Morton 1953) have observed that the velocities of bubbles in the size range $0.3-3.0 \mathrm{~mm}$ 
are reduced somewhat if the water is impure or contains 'surface-active' substances. However, Haberman \& Morton report that air bubbles in filtered tap water rise with the same velocities as bubbles in distilled water.

The theoretical work so far published has been confined to bubbles which are either so small or rise so slowly that the approximations of the slow viscous motion theory can be applied, or to large bubbles which are in the form of spherical caps (see Davies \& Taylor 1950). It is the object of this paper to give a theoretical account of some aspects of the motion of the spiralling and zig-zagging bubbles.

In $\S 3$ we consider the spiralling bubbles. The method used is briefly as follows. We suppose that the motion of the water near the front of the bubble is inviscid and irrotational, and calculate it in terms of the velocity of rise, the shape and orientation of the bubble, and the dimensions of the spiral. We can then find the pressure at the front surface of the bubble. The pressure inside the bubble is the sum of the pressure outside and a term involving the surface tension and curvature of the surface. We may take this sum to be constant at all points of the surface, since the pressure variations inside the bubble can be neglected owing to the small density of air compared with that of water. We apply this boundary condition and obtain relations between the quantities mentioned above; in particular we obtain an equation giving the velocity of rise of a spiralling bubble in terms of its size. It is to be noted that this method does not require a knowledge of the boundary condition to be satisfied by the velocity of the fluid at the surface of the bubble, since it is assumed that the effect of viscosity on the motion at the front of the bubble is confined to a thin region.

This method is used in $\$ 4$ to investigate the motion of a zig-zagging bubble. In this case, we find that instead of obtaining an equation involving the velocity of rise and the bubble size, a stability equation results from the analysis. This equation shows that the motion near the front of a bubble which is rising steadily in a straight line is unstable when the bubble is sufficiently oblate.

To obtain further information about the motion, it is necessary to consider properties of the wake behind the bubble. There is some relevant evidence on the wake behind solid bodies, and this is discussed in $\S 4$ in connection with the zig-zag motion.

\section{EXPERIMENTAL METHOD AND RESULTS}

Air bubbles were observed as they rose up through a rectangular tank of height 15 in. and cross-section $12 \times 12$ in., which was filled with either filtered (standard No. 1 filter papers were used) or unfiltered tap water at temperatures between $16^{\circ} \mathrm{C}$ and $19^{\circ} \mathrm{C}$. They were released at the bottom of the tank from the end of a capillary tube, and were introduced into the other end of the tube by squeezing a rubber syringe containing air (see figure 1). 
The experimental procedure was as follows. Tap ' $A$ ' was opened, and tap ' $B$ ' was so adjusted that water flowed very slowly from the T-shaped glass tube through the capillary tube into the tank. Then tap 'A' was closed, and the rubber syringe containing air was squeezed gently until an air bubble of about the size required formed in the funnel-shaped end of the capillary tube. Tap ' $A$ ' was opened again, and the air bubble rose up along the capillary tube until it came opposite to the scale. Tap 'A' was then closed; the bubble stopped moving, and its length was measured on the scale. Since the bore of the tube had been found accurately by the usual methods, the volume of the bubble could be calculated with sufficient accuracy. Tap 'A' was now opened again and kept open until the bubble reached the end of the capillary tube at the bottom of the tank, whereupon the tap was closed so that the water in the tank could come to rest. 'Tap 'A' was opened once again, and the bubble rose up through the tank and was observed either through the sides of the tank or from above. Once sufficient skill in making the delicate adjustments had been acquired, the method worked very well, and it was possible to obtain bubbles of about the desired size at will. With this arrangement it was possible, by adjusting the tap ' $B$ ', to vary the pressure in the T-shaped tube in such a way that bubbles in the capillary tube could be moved, with adjustable speed, in either direction.

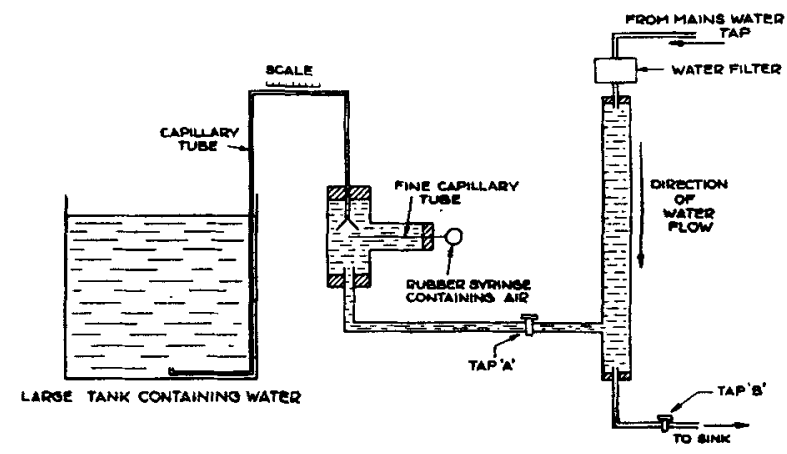

Figure 1. Experimental apparatus (not to scale).

Bubbles of radii between 0.5 and $2.3 \mathrm{~mm}$ were observed during the course of the experiments, a capillary tube of bore $0.8 \mathrm{~mm}$ being used for the smaller bubbles and one of bore $1.6 \mathrm{~mm}$ for the larger. Bubbles of radius larger than $2.3 \mathrm{~mm}$ could not be produced since they always broke up before leaving the capillary tube, even when tubes of larger bore were used. In fact, great care was needed in order to make bubbles of radius larger than about $2.0 \mathrm{~mm}$. The following observations were made on bubbles in filtered water.

If the radius was less than $0.7 \mathrm{~mm}$, the bubble rose steadily in a straight line. If the radius was between 0.7 and $1.0 \mathrm{~mm}$, the motion was a zig-zag, i.e. a side-to-side movement in a plane; the direction of the plane remained 
constant as the bubble was rising, but there was no apparent tendency for the bubbles to prefer any particular plane.

When the radius was between 1.0 and $2.3 \mathrm{~mm}$, the situation was more complicated. It was found that bubbles in this size range would either spiral or zig-zag, depending upon various factors. For instance, it sometimes happened that a large bubble would break up into two parts just before it left the capillary tube. The first part would rise through the tank and always spiral, whilst the other stayed in the capillary tube. The size of the part left behind could be measured by bringing it back to the scale; and, since the size of the original bubble had been measured, the size of the spiralling bubble was known. When this break-up occurred, the radius of the bubble that left the tube was always greater than $1.3 \mathrm{~mm}$; it was not possible to produce this break-up at will and smaller bubbles could not be produced in this manner.

However, if care was taken to see that external disturbances were reduced to a minimum and the bubble left the capillary tube in one piece, then the bubble would usually zig-zag. A bubble could usually be made to spiral by 'hitting' it, i.e. by placing an obstacle above the end of the capillary tube and in the path of the bubble. Further, if two bubbles were released shortly after one another, the second would spiral if the first one did; but if the first one zig-zagged, then so usually did the second. It was noticed that a zig-zag would sometimes change into a spiral, but the opposite was never observed. Bubbles of radius less than $1.0 \mathrm{~mm}$ were always observed to zig-zag, even after being 'hit' or released in the wake of a spiralling bubble. Bubbles were also projected at speed from the capillary tube, but there appeared to be no correlation between the initial velocity of the bubble and the occurrence of zig-zag or spiral motion.

The general impression received from these observations was that, when the radius was greater than $1.0 \mathrm{~mm}$, the zig-zag motion was unstable to sufficiently large disturbances and the bubble would spiral, the tendency to spiral increasing as the size increased.

Furthermore, bubbles of radius about $0.7 \mathrm{~mm}$ were sometimes observed to rise steadily, then zig-zag, then go straight again, zig-zag again, and so on. When bubbles of this size were released in the wake of a spiralling or zig-zagging bubble, they would zig-zag with the usual frequency, but ' hitting' these bubbles would not produce a regular zig-zag. Slightly smaller bubbles of radius about $0.6 \mathrm{~mm}$ were also 'hit' and released in the wake of a larger bubble, but they always rose steadily in a straight line.

Similar experiments were carried out using unfiltered tap water. The results were the same except that the zig-zag motion first occurred at a radius of $0.8 \mathrm{~mm}$, and that it was not possible to produce the spiralling motion when the radius was less than $1.1 \mathrm{~mm}$.

To sum up, these experiments suggest strongly that when the bubble radius reaches the value at which the steady rise in a straight line becomes unstable, the zig-zag motion appears first; and that at a slightly larger radius, this zig-zag motion is itself unstable to sufficiently large disturbances, 
and a spiralling motion appears. The observations that the zig-zag motion occurs first and sometimes changes into the spiralling motion, whereas the spiralling motion never changes into the zig-zag motion, indicate that the spiralling motion arises from a later instability than the zig-zag motion. However, some authors (e.g. Miyagi 1925) claim to have observed only spiralling bubbles, and it seems likely that under certain experimental conditions, zig-zagging does not occur for any bubble size. These conditions will presumably be related to the way in which the bubbles are produced, and it is possible that, in such cases, the disturbances that are present are sufficiently strong to make the zig-zag motion always unstable.

\section{The motion of a spiralling bubble}

\section{The formulation of the problem}

We shall now give a theoretical treatment of the motion of a spiralling bubble. The problem is complicated, and we shall make several simplifying assumptions in order to render it tractable. One complication is the need to determine the shape of the bubble. It can be shown that a bubble is nearly spherical, when the Reynolds number is large, if $2 g R / W^{2}$ (the inverse of the Froude number) and $2 \rho R W^{2} / T$ (the Weber number) are both much less than one. Here, $T$ is the surface tension, $R$ the bubble radius, $W$ the velocity of rise, and $\rho$ the density of the fluid. For spiralling (and zig-zagging) bubbles $2 g R / W^{2}$ is small but $2 \rho R W^{2} / T$ is of the order of unity; consequently, we cannot suppose the bubble to be nearly spherical and make use of the simplifications that are then possible. This conclusion is consistent with the experimental observation (see, for example, Rosenberg 1950) that spiralling bubbles are not spherical but are approximately oblate spheroids. It will be assumed, for the purposes of the analysis, that the bubble is an oblate spheroid. This is not strictly true, but it seems to be a reasonable approximation.

The spiral along which the bubble moves appears from the experiments to be roughly uniform and to be described at a constant rate. We shall therefore suppose that the motion is steady when referred to axes fixed in the bubble, and that the shape of the bubble is constant. We can, in fact, imagine the bubble to be at rest and the water to be streaming past it steadily. In order to simplify the analysis further, it will also be supposed that the angle of the spiral (which is defined as the angle between the upward vertical and the tangent to the spiral) is small, and we shall neglect, in the subsequent equations, terms that are of the same order of magnitude as this angle. Later, we shall estimate the numerical magnitude of such terms and see that this approximation is reasonable. It will also be supposed that the angle between the axis of symmetry and velocity of the bubble is small compared with the angle of the spiral; and it then seems permissible, for the sake of simplicity, to take the axis of symmetry coplanar with the velocity of the bubble and the vertical, i.e. to suppose that the axis of symmetry lies in the tangential plane of the cylinder that contains the spiral. This 
assumption is consistent with the rough observations made by several authors (e.g. Miyagi 1925) that the axis of symmetry and velocity of the bubble are parallel, and can also be shown to be self-consistent with the results of the analysis given below.

We have now set up the geometry of the problem, and we shall now consider how we may calculate the pressure $p$ in the water. The basic assumption, upon which the analysis in this paper is based, is that the pressure over the surface of the bubble near the front stagnation point is the same as if the whole flow were inviscid and irrotational. In effect, we assume here that the motion near the front of the bubble is inviscid and that the velocity and pressure near the front stagnation point are primarily determined by the shape of the neighbouring surface. Now the boundary condition to be satisfied by the pressure is (neglecting pressure variation inside the bubble)

$$
p+T\left(1 / R_{1}+1 / R_{2}\right)=\text { const. }
$$

over the whole surface of the bubble, where $R_{1}$ and $R_{2}$ are the principal radii of curvature of the surface. We then make use of the above assumption by supposing that the first and second derivatives of equation (1), differentiating along the surface, are zero at the front stagnation point when the pressure calculated on the assumption of ideal flow is substituted for $p$. From the equations so obtained, we shall derive equations relating the geometrical parameters of the spiral, the shape of the bubble and the velocity of rise.

It is worth noting that Davies \& Taylor, making assumptions about the pressure that are basically the same as those made here, calculated the velocity of rise of large spherical-cap bubbles, and the theoretical velocities agreed well with the velocities which they observed experimentally. The Reynolds number for these large bubbles was several thousand. The Reynolds numbers of the spiralling bubbles dealt with here are several hundred, which should still be sufficiently large to justify the assumption that flow over the front of the bubble is essentially inviscid. We shall, however, modify later the assumption of ideal flow in order to take account of possible separation of the flow around the bubble. In this way, we shall obtain results in agreement with the observations of bubbles rising in impure water. This point will be discussed again in more detail.

It should be noted that the theoretical treatment given here is incomplete in the sense that we do not investigate conditions at the rear of the bubble, owing to the extreme complexity of the motion there. The consequences of this incompleteness are that we shall obtain fewer equations than unknowns, and that we shall obtain no information as to the causes of the spiralling motion.

\section{The calculation of the pressure}

We denote the vertical velocity of rise by $W$, the angular velocity with which the spiral is described by $\Omega$, and the radius of the spiral by $d$. 
We take axes $(x, y, z)$ with origin fixed at the centre of the bubble; $x$ is the axis of symmetry, and the plane $x, y$ contains the vertical.

Further, we take oblate spheroidal coordinates $\mu, \zeta, \omega$ defined by $x=k \mu \zeta, y=k\left(1-\mu^{2}\right)^{1 / 2}\left(1+\zeta^{2}\right)^{1 / 2} \cos \omega, z=k\left(1-\mu^{2}\right)^{1 / 2}\left(1+\zeta^{2}\right)^{1 / 2} \sin \omega($ see, for example, Lamb (1932) §107). We suppose that the surface of the bubble corresponds to $\zeta=\zeta_{0}$, so that the eccentricity of a meridian plane of the bubble is $\left(1+\zeta_{0}^{2}\right)^{-1 / 2}$, and the equivalent spherical radius is $k\left\{\zeta_{0}\left(1+\zeta_{0}^{2}\right)\right\}^{1 / 3}$. We denote by $\chi$ the angle between the axis of symmetry of the bubble and the upward vertical.

We now reduce the bubble to rest by superposing a velocity $-W$ and an angular velocity $-\Omega$ on the fluid, where $\mathbf{W}=(W \cos \chi, W \sin x, 0)$ and $\Omega=(\Omega \cos \chi, \Omega \sin \chi, 0)$. The velocity of the undisturbed fluid is now $-\mathbf{W}-\boldsymbol{\Omega} \times(\mathbf{d}+\mathbf{r})$, where $\mathbf{d}=(0,0, d)$, and $\mathbf{r}=(x, y, z)$. Let us denote by $\Phi$ the velocity potential of the disturbance due to the bubble, so that $\mathbf{V}=\nabla \Phi-\mathbf{W}-\mathbf{\Omega} \times(\mathbf{d}+\mathbf{r})$ is the velocity of the fluid relative to the axes $(x, y, z)$. (The actual velocity of the fluid relative to axes fixed in space is, of course, just $\nabla \Phi$.)

The surface of the bubble is now a streamline, so that $\mathbf{V} . \mathbf{n}=0$ on $\zeta=\zeta_{0}$, where $\mathbf{n}$ is the outward normal to the surface. Hence, after some algebra, we obtain

$$
\begin{aligned}
\left(\frac{\partial \Phi}{\partial \zeta}\right)_{\zeta=\zeta_{0}}= & \mu k(W \cos \chi+\Omega d \sin \chi)+ \\
& +k\left(1-\mu^{2}\right)^{1 / 2}\left(1+\zeta_{0}^{2}\right)^{-1 / 2} \zeta_{0}(W \sin \chi-\Omega d \cos \chi) \cos \omega+ \\
& \quad+\Omega k^{2} \mu\left(1-\mu^{2}\right)^{1 / 2}\left(1+\zeta_{0}^{2}\right)^{-1 / 2} \sin \chi \sin \omega .
\end{aligned}
$$

We are supposing the fluid to be incompressible, so that $\Phi$ satisfies $\nabla^{2} \Phi=0$. Suitable solutions of this equation which fit the boundary condition (2) and which vanish at infinity are

$$
\begin{gathered}
\mu\left(1-\cot \zeta^{-1} \zeta\right), \quad\left(1-\mu^{2}\right)^{1 / 2}\left(1+\zeta^{2}\right)^{-1 / 2}\left[\zeta-\left(1+\zeta^{2}\right) \cot ^{-1} \zeta\right] \cos \omega \\
\mu\left(1-\mu^{2}\right)^{1 / 2}\left(1+\zeta^{2}\right)^{1 / 2}\left[3 \zeta \cot ^{-1} \zeta-3+\left(1+\zeta^{2}\right)^{-1}\right] \sin \omega .
\end{gathered}
$$

We then find, after some algebra, that on $\zeta=\zeta_{0}$,

$$
\begin{gathered}
\Phi=-k \mu\left(1+\zeta^{2}\right)^{2}\left(1-\zeta \cot ^{-1} \zeta\right)(W \cos \chi+\Omega d \sin \chi) Z^{-1}- \\
-k Z Y \zeta\left(1+\zeta^{2}\right)^{-1 / 2}(W \sin \chi-\Omega d \cos \chi)\left(1-\mu^{2}\right)^{1 / 2} \cos \omega+ \\
+\Omega k^{2} \sin \chi\left(\zeta^{2}+1\right)^{1 / 2}(X-\zeta) \mu\left(1-\mu^{2}\right)^{1 / 2} \sin \omega
\end{gathered}
$$

where for brevity we write

$$
\begin{aligned}
& X=\zeta+\frac{3 \zeta \cot ^{-1} \zeta-3+\left(1+\zeta^{2}\right)^{-1}}{\left(6 \zeta^{2}+3\right) \cot ^{-1} \zeta-6 \zeta-\zeta\left(1+\zeta^{2}\right)^{-1}}, \\
& Y=\left\{2+\zeta^{2}-\zeta\left(1+\zeta^{2}\right) \cot ^{-1} \zeta\right\}^{-1}, \\
& Z=\left(1+\zeta^{2}\right)\left\{\left(1+\zeta^{2}\right) \cot ^{-1} \zeta-\zeta\right\},
\end{aligned}
$$

and the suffix of $\zeta_{0}$ has been dropped.

Let $V_{\mu}$ and $V_{\omega}$ denote the components of $\mathbf{V}$ on the surface of the bubble in the directions of increasing $\mu$ and $\omega$ respectively; $V_{\zeta}$ is, of course, zero. 
We use (3) and obtain, after some algebra,

and

$$
\begin{aligned}
\left(\mu^{2}+\zeta^{2}\right)^{1 / 2} V_{\mu}= & -Z^{-1}\left(1+\zeta^{2}\right)(W \cos \chi+\Omega d \sin \chi)\left(1-\mu^{2}\right)^{1 / 2}+ \\
& +2 Y\left(1+\zeta^{2}\right)^{1 / 2}(W \sin \chi-\Omega d \cos \chi) \mu \cos \omega+ \\
& +\Omega k \sin \chi\left(1+\zeta^{2}\right)^{1 / 2}\left[-\zeta+\left(1-2 \mu^{2}\right)(X-\zeta)\right] \sin \omega
\end{aligned}
$$

$$
\begin{aligned}
V_{\omega}= & 2 Y(W \sin \chi-\Omega d \cos \chi) \sin \omega- \\
& -\Omega k \cos \chi\left(1+\zeta^{2}\right)^{1 / 2}\left(1-\mu^{2}\right)^{1 / 2}+\Omega k \sin \chi X \mu \cos \omega .
\end{aligned}
$$

At the front stagnation point, $V_{\mu}$ and $V_{\omega}$ are zero, and the coordinates of the stagnation point, $\left(\mu_{s}, \omega_{s}\right)$ say, are given by putting the expressions (4) and (5) equal to zero.

The pressure is then given by*

$$
p / \rho+\frac{1}{2} \mathbf{V}^{2}+g H-\frac{1}{2} \Omega^{2} \varpi^{2}=\text { const. }
$$

on a streamline, where $g$ is the acceleration due to gravity,

$$
\varpi=\left[(d+z)^{2}+(x \sin \chi-y \cos \chi)^{2}\right]^{1 / 2}
$$

denotes the perpendicular distance from the axis of the spiral and

$$
H=x \cos \chi+y \sin \chi
$$

is the vertical height above the centre of the bubble. Further,

$$
\frac{1}{R_{1}}+\frac{1}{R_{2}}=\frac{\zeta\left(\zeta^{2}+1\right)^{1 / 2}}{k\left(\zeta^{2}+\mu^{2}\right)^{3 / 2}}+\frac{\zeta}{k\left(\zeta^{2}+1\right)^{1 / 2}\left(\zeta^{2}+\mu^{2}\right)^{1 / 2}}
$$

by well-known results of solid geometry.

\section{The application of the pressure boundary condition}

Now the surface of the bubble is a streamline and so, in view of the initial assumption about the pressure, the boundary condition is

$$
\left(\frac{\partial}{\partial \mu}, \frac{\partial}{\partial \omega}, \frac{\partial^{2}}{\partial \mu^{2}}, \frac{\partial^{2}}{\partial \mu \partial \omega}, \frac{\partial^{2}}{\partial \omega^{2}}\right)\left(-\frac{1}{2} \mathbf{V}^{2}-g H+\frac{1}{2} \Omega^{2} w^{2}+\frac{T}{\rho}\left(\frac{1}{R_{1}}+\frac{1}{R_{2}}\right)\right)=0,
$$

the derivatives being evaluated at the stagnation point $\left(\mu_{s}, \omega_{s}\right)$.

From (6), $\partial / \partial \mu$ gives

$$
\begin{aligned}
& -\frac{T \zeta \mu\left(4 \zeta^{2}+\mu^{2}+3\right)}{\rho k\left(\zeta^{2}+\mu^{2}\right)^{5 / 2}\left(1+\zeta^{2}\right)^{1 / 2}}-g k \zeta \cos \chi+g k \sin \chi\left(1+\zeta^{2}\right)^{1 / 2} \mu\left(1-\mu^{2}\right)^{-1 / 2} \cos \omega- \\
& -\Omega^{2} k d\left(1+\zeta^{2}\right)^{1 / 2} \mu\left(1-\mu^{2}\right)^{-1 / 2} \sin \omega+ \\
& +\Omega^{2} k^{2}\left\{\mu \zeta^{2} \sin ^{2} \chi-\mu\left(1+\zeta^{2}\right)\left(\sin ^{2} \omega+\cos ^{2} \chi \cos ^{2} \omega\right)+\right. \\
& \left.\quad+\left(2 \mu^{2}-1\right) \zeta\left(1+\zeta^{2}\right)^{1 / 2} \sin \chi \cos \chi\left(1-\mu^{2}\right)^{-1 / 2} \cos \omega\right\}=0 ; \quad \text { (7) }
\end{aligned}
$$

*This equation is the appropriate form of Bernoulli's equation for steady motion relative to moving and rotating axes. One way of deducing it is as follows. The pressure is given by $p / \rho+\frac{1}{2}(\nabla \Phi)^{2}+\partial \Phi / \partial t+g H=$ constant, where $\partial / \partial t$ denotes differentiation with respect to axes fixed in space. The velocity in space of the axes $(x, y, z)$ is $\mathbf{W}+\Omega \times \mathbf{d}$ and the angular velocity of these axes is $\Omega$; and it follows that $\partial \Phi / \partial t=-[\mathbf{W}+\mathbf{\Omega} \times(\mathbf{d}+\mathbf{r})] . \nabla \Phi$. Remembering that $\nabla \Phi=\mathbf{V}+\mathbf{W}+\mathbf{\Omega} \times(\mathbf{d}+\mathbf{r})$ we obtain the given equation on substitution. 
and $\partial / \partial \omega$ gives

$k\left(1+\zeta^{2}\right)^{1 / 2}\left(1-\mu^{2}\right)^{1 / 2}\left\{g \sin \chi \sin \omega+\Omega^{2} d \cos \omega+\right.$

$\left.+\Omega^{2} k \sin ^{2} \chi\left(1+\zeta^{2}\right)^{1 / 2}\left(1-\mu^{2}\right)^{1 / 2} \sin \omega \cos \omega+\Omega^{2} k \sin \chi \cos \chi \zeta \mu \sin \omega\right\}=0,(8)$

the suffixes of $\mu_{s}$ and $\omega_{s}$ having been dropped since all expressions are evaluated at the stagnation point.

We now suppose that powers of $\chi$ and $\left(1-\mu^{2}\right)^{1 / 2}$ higher than the first can be neglected. It follows from equations (12) and $\left(4^{\prime}\right)$ obtained below that these quantities are of the same order of magnitude as the angle of the spiral, which is supposed to be small. Equations (4), (5), (7) and (8) thus become

$$
\begin{gathered}
W Z^{-1}\left(1+\zeta^{2}\right)^{1 / 2}\left(1-\mu^{2}\right)^{1 / 2}+2 Y \cos \omega(W \chi-\Omega d)-\Omega k X \chi \sin \omega=0, \\
-\Omega k\left(1+\zeta^{2}\right)^{1 / 2}\left(1-\mu^{2}\right)^{1 / 2}+2 Y \sin \omega(W \chi-\Omega d)+\Omega k X \chi \cos \omega=0, \\
\left\{\frac{4 T \zeta}{\rho g k^{2}\left(1+\zeta^{2}\right)^{2}}+\zeta+\frac{\Omega^{2} k}{g}\left(1+\zeta^{2}\right)\right\}\left(\frac{1-\mu^{2}}{1+\zeta^{2}}\right)^{1 / 2}+\frac{\Omega^{2} d}{g} \sin \omega- \\
-\left(1+\frac{\Omega^{2} k \zeta}{g}\right) \times \cos \omega=0, \\
\left(1+\frac{\Omega^{2} k \zeta}{g}\right) \chi \sin \omega+\frac{\Omega^{2} d}{g} \cos \omega=0 .
\end{gathered}
$$

and

We now eliminate $\mu$ and $\omega$ from these four equations and obtain

and

$$
2 Y\left(W-\frac{\Omega d}{\chi}\right)\left(1-\frac{k g Z_{\chi}}{\Omega d W}\right)=\frac{g k X_{\chi}}{\Omega d},
$$

$$
\begin{aligned}
& \frac{W}{Z}\left(1+\zeta^{2}\right)\left(\chi^{2}+\frac{\Omega^{4} d^{2}}{g^{2}}\right)^{1 / 2} \\
& \quad=\frac{g k X \chi}{\Omega d}\left(\frac{4 T \zeta}{\rho g k^{2}\left(1+\zeta^{2}\right)^{2}}+\zeta\right)\left[\frac{\Omega^{4} d^{2}}{g^{2}}+\chi^{2}\left(1-\frac{k g Z \chi}{\Omega d W}\right)^{-2}\right]^{1 / 2},
\end{aligned}
$$

where we have neglected terms of order $\Omega^{2} k \zeta / g$ and $\Omega^{2} k^{2} / W^{2}$, which can be shown to be of the same order of magnitude as the angle of the spiral.

Let us now consider the second order derivatives in (6), using again the assumptions that $\chi$ and $\left(1-\mu^{2}\right)^{1 / 2}$ are small. From $\partial^{2} / \partial \mu^{2}$ we obtain

$$
\frac{W^{2}}{g k}=\frac{Z^{2}}{1+\zeta^{2}}\left\{\frac{4 T \zeta}{\rho g k^{2}\left(1+\zeta^{2}\right)^{2}}+\zeta\right\} ;
$$

from $\partial^{2} / \partial \mu \partial \omega$ we obtain a combination of equations $\left(4^{\prime}\right),\left(5^{\prime}\right)$ and $\left(8^{\prime}\right)$; and from $\partial^{2} / \partial \omega^{2}$ we obtain a combination of equations (11) and $\left(7^{\prime}\right)$.

Thus, we have obtained three complicated equations, namely (9), (10) and (11), which give the five unknowns $W, \Omega, d, \zeta, \chi$ in terms of $T / \rho, g$ and the equivalent spherical radius $r\left(r\right.$ and $k$ are related by $\left.r^{3}=k^{3} \zeta\left(1+\zeta^{2}\right)\right)$. These equations have been derived from an analysis of the motion at the front of the bubble; and it is not surprising that we should obtain fewer equations than unknowns since we have considered only one region of the flow. Other equations would presumably result from a study of the motion in the wake and the forces acting on the bubble.

However, we can simplify these equations and determine $\zeta$ and the velocity of rise provided that we neglect $k g Z_{\chi} /(\Omega d W)$. Later, we shall 
make an estimate of this quantity and see that its neglect is reasonable for bubbles whose radius is less than $1.5 \mathrm{~mm}$, but not for larger ones. Equations (9) and (10) then become

and

$$
2 Y\left(W-\frac{\Omega d}{\chi}\right) \frac{\Omega d}{\chi}=k g X
$$

$$
\left(1+\zeta^{2}\right) \frac{W}{Z}=\frac{g k X \chi}{\Omega d}\left(\frac{4 T \zeta}{\rho g k^{2}\left(1+\zeta^{2}\right)^{2}}+\zeta\right)
$$

Combining $\left(10^{\prime}\right)$ and $(11)$, we obtain

$$
\frac{\Omega d}{W_{\chi}}=\frac{X}{Z}
$$

and from (12), $\left(9^{\prime}\right)$ and (11), we then obtain

$$
\frac{4 T}{\rho g k^{2}\left(1+\zeta^{2}\right)^{2}}=\frac{1+\zeta^{2}}{2 Y \zeta(Z-X)}-1
$$

Equation (13) determines the shape parameter $\zeta$ as a complicated function of the bubble radius, and then the velocity of rise follows from equation (11). Equation (12) contains the remaining three unknowns, and gives the ratio of the angles made with the vertical by the velocity and axis of symmetry of the bubble.

\section{Comparison with experiment}

We shall now discuss the above theoretical results and compare them with the experimental observations of various authors. It will be seen that the agreement between the velocity of rise and bubble shape predicted by equations (11) and (13) and the experimental observations is reasonable, considering the nature of the approximations that have been made. We. shall then examine some of the approximations in more detail and see how they might account for some of the disagreement between theory and experiment.

Figure 2 shows how $X, Y^{-1}$ and $Z$ vary with $\zeta$. It will be noticed that $Z$ is greater than $X$ only if $\zeta$ is less than $1 \cdot 52$. Hence, the right-hand side of equation (13) is positive, which is obviously necessary if $r$ is to be real, only if the bubble is sufficiently flattened (it will be remembered that the smaller $\zeta$, the less spherical is the bubble). In other words, no bubble can spiral unless it is flatter than the oblate spheroid corresponding to $\zeta=1 \cdot 52$.

In figure 3 is shown the solution of equation (13) for air bubbles in water, where the typical values $T / \rho=74 \mathrm{~cm}^{3} \mathrm{sec}^{-2}$ and $g=981 \mathrm{~cm} \mathrm{sec}^{-2}$ have been used. For each value of $r$ less than $3.6 \mathrm{~mm}$, there are two values of $\zeta$; and we take the larger value as the one corresponding to physical reality, since then $\zeta$ decreases as $r$ increases. (Surface tension tends to keep the bubble spherical, and the magnitude of the surface tension effect is inversely proportional to the radius of the bubble.)

Further, there are no values of $\zeta$ which correspond to $r$ greater than $3.6 \mathrm{~mm}$, and we can interpret this as meaning that the spiralling motion is not possible for bubbles of radius larger than $3.6 \mathrm{~mm}$. This agrees 


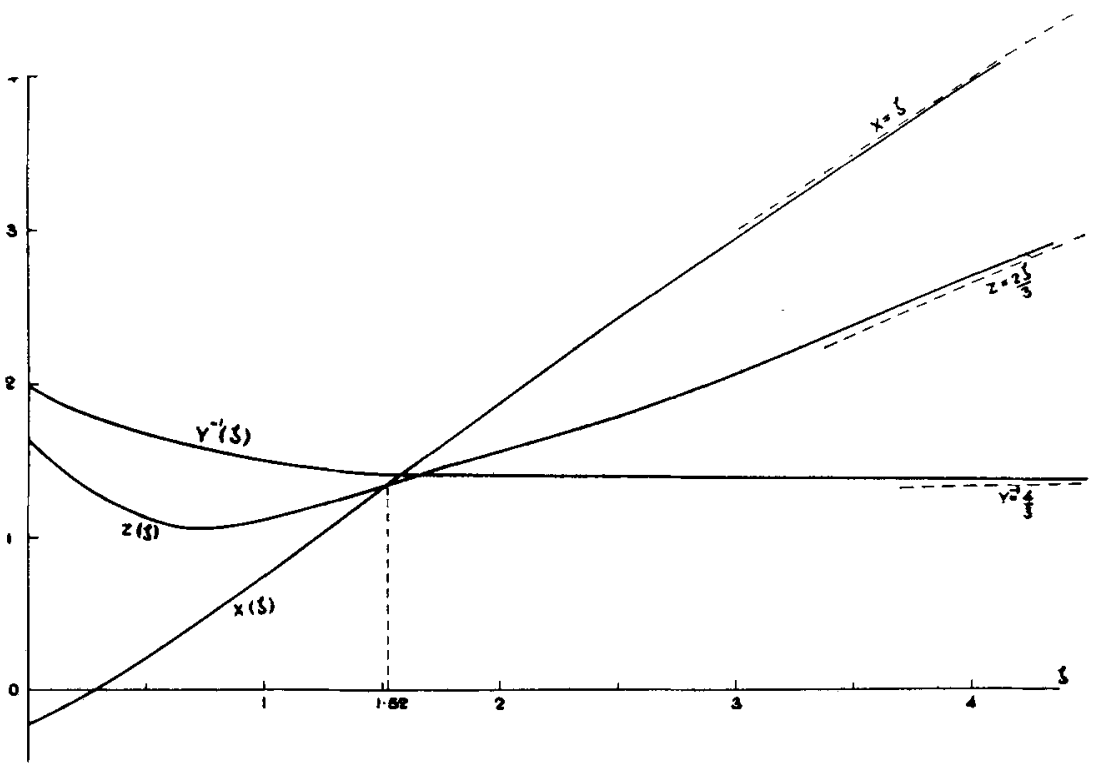

Figure 2. $X, Y^{-1}$ and $Z$ as functions of $\zeta$.

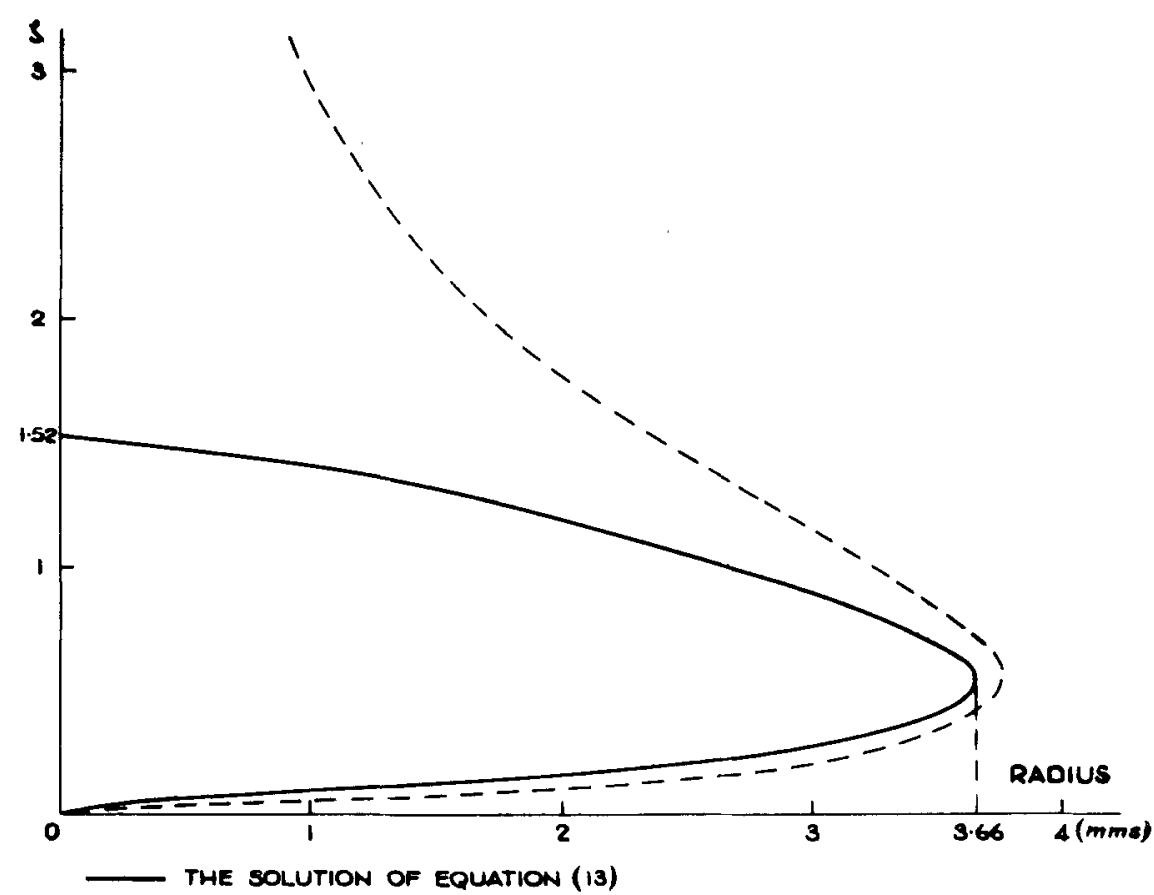

AN EXAMPLE OF HOW THE SOLUTION MAY BE MODIFIEO BY SEPARATION OF THE FLOW. SOLUTION OF EQUATION (13)" WITH $K=1.75$

Figure 3. $\zeta$ as a function of the bubble radius. 
qualitatively with the experimental observations of the gradual disappearance of the spiralling motion as the radius of the bubble increases. For example, Rosenberg (1950) claims that the spiralling motion ceases when the radius becomes equal to $2: 4 \mathrm{~mm}$. We shall see below that there are indications that the figure of $3.6 \mathrm{~mm}$ would be reduced somewhat if $k g Z_{\chi} /(W \Omega d)$ were not neglected.

The ratio $a / b$, as given by the analysis, of the longest to the shortest axis of the bubble is plotted against the radius in figure 4 . The experimental measurements of this ratio by Rosenberg (1950) are also shown. The agreement is not too good, especially for the larger bubbles, but we shall see that the neglect of $k g Z_{\chi} /(W \Omega d)$ leads to the value of $a / b$ for the larger bubbles being somewhat too small. Another reason for the discrepancy may be the assumption that the bubble is exactly an oblate spheroid.

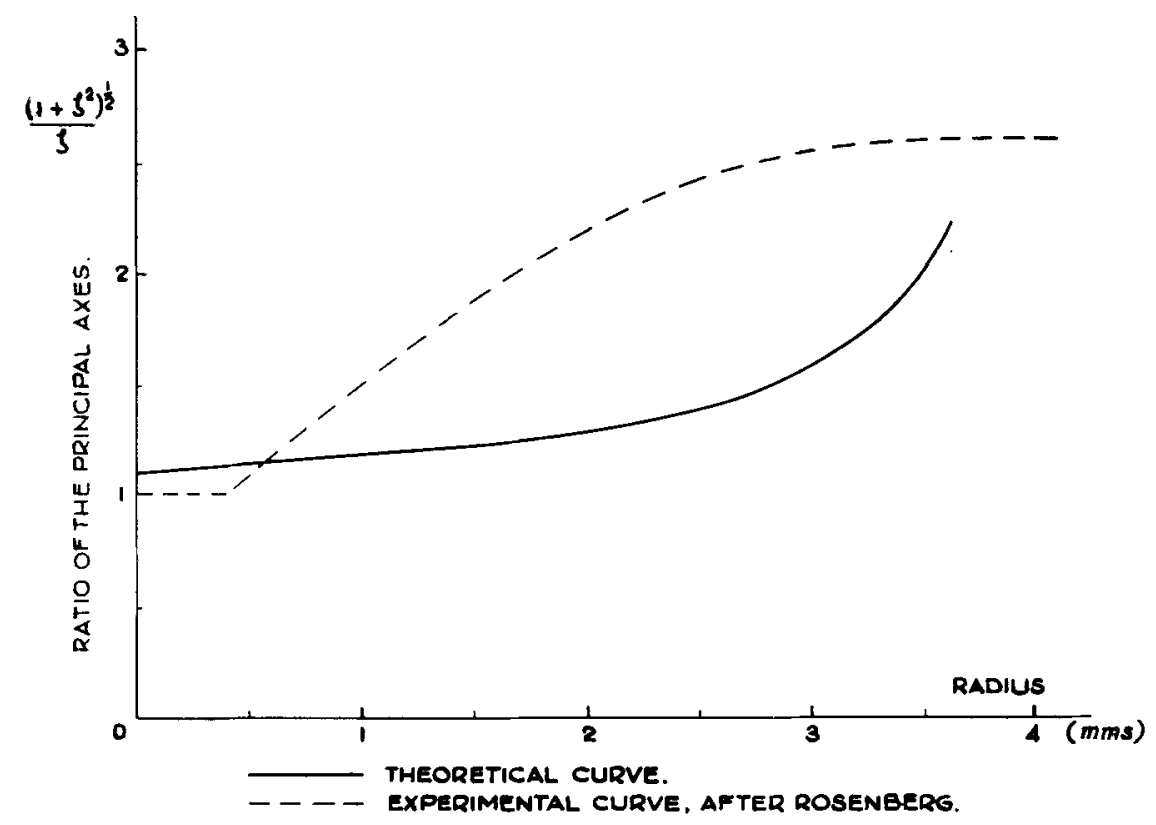

Figure 4. The shape of air bubbles.

The velocity of rise as given by equations (11) and (13) is shown in figure 5, together with experimental results for spiralling bubbles given by Datta, Napier \& Newitt (1950) and Haberman \& Morton (1953). The theoretical and experimental results are in qualitative agreement but the theoretical values are about $20 \%$ too small. The theoretical velocity goes off to infinity as the radius decreases, but small bubbles do not spiral and the analysis would therefore not apply to them. We shall discuss below reasons why the calculated velocities are too small.

We see from equation (12) that $\Omega d /\left(W_{\chi}\right)$ decreases as $r$ increases, being one when $r$ is zero, about 0.75 when $r=2.3 \mathrm{~mm}$, and about 0.2 when 
$r=3.6 \mathrm{~mm}$. Provided that the bubble radius is not too large, this result is consistent with the rough experimental observations that $\Omega d /(W \chi)=1$ (see, for example, Miyagi (1925)). (It follows from $\left(12^{\prime}\right)$ below that for large bubbles $\Omega d /\left(W_{\chi}\right)$, as predicted by (12), is too small.)

Let us now consider the magnitude of some of the terms that were neglected during the course of the analysis. First, there are assumptions that certain geometrical quantities, such as the angle of the spiral $\Omega d / W$ and $\chi$, are small. With typical values such as $\Omega=30$ radians $/ \mathrm{sec}$, $d=1.5 \mathrm{~mm}$ and $W=30 \mathrm{~cm} / \mathrm{sec}$, it is easily seen that all such geometrical quantities that have been neglected could possibly account for the discrepancies between theory and experiment, but this does not seem likely.

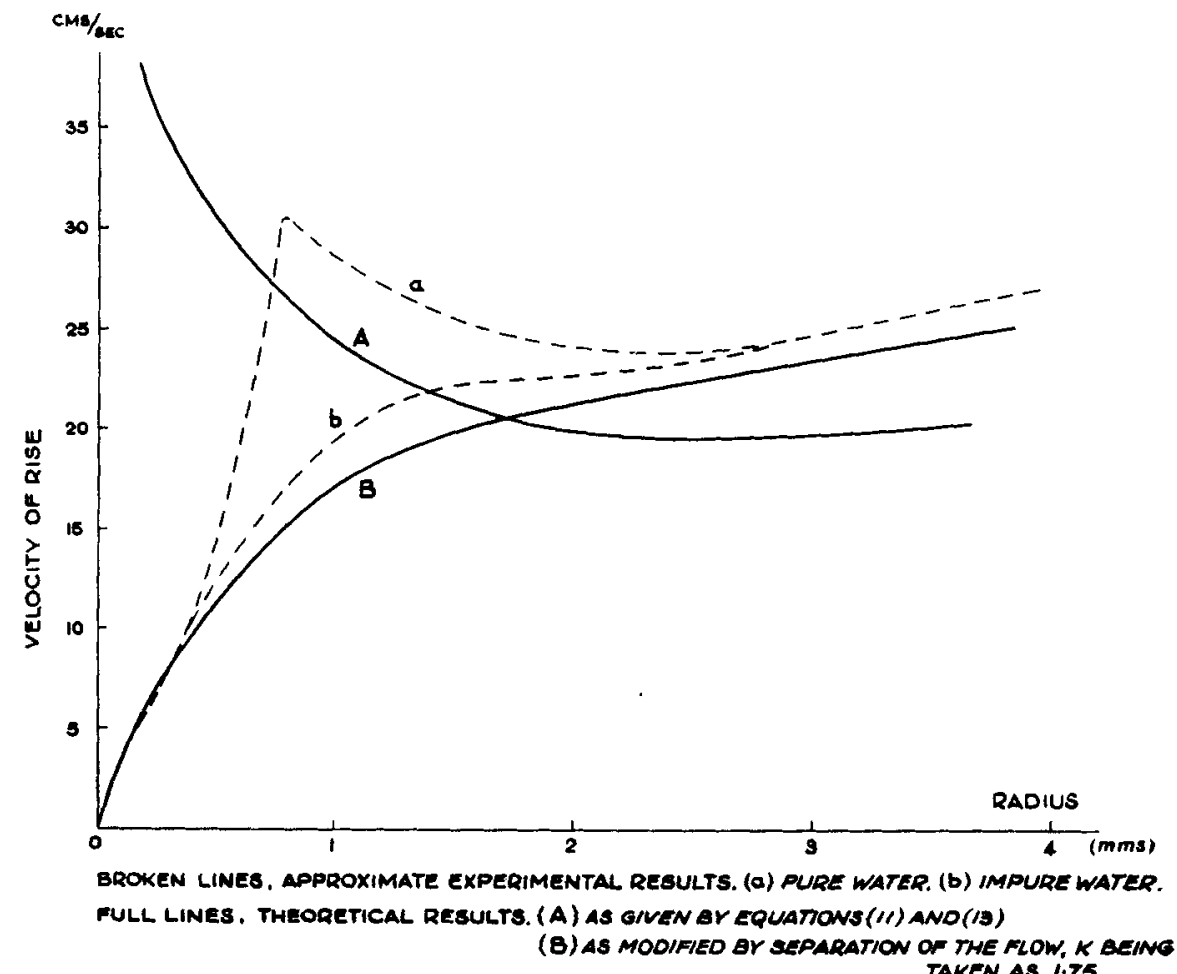

Figure 5. The velocity of rise of spiralling bubbles.

Of more importance is the neglect of $k g Z_{\chi} /(W \Omega d)=\Lambda$, say. (The physical meaning of $\Lambda$ is not altogether clear, although the requirement that it is small probably expresses the condition that variations of hydrostatic pressure over the bubble surface are small compared with those of the hydrodynamic pressure.) When $r$ is less than $1.5 \mathrm{~mm}, \Lambda$ is less than 0.25 , and its neglect is reasonable. However, if we determine $\chi$ by equation (12), $\Lambda$ increases rapidly with $r$ and is of the order of unity when the radius is about $3.0 \mathrm{~mm}$; therefore equations (12) and (13) will become more and more inaccurate as the bubble becomes larger. 
If we did not neglect $\Lambda$, equations (12) and (13) would be

and

$$
\frac{\Omega d}{W_{\chi}}=\frac{X^{*}}{Z}
$$

where

$$
\frac{4 T}{\rho g k^{2}\left(1+\zeta^{2}\right)^{2}}=\frac{\left(1+\zeta^{2}\right) X}{2 Y \zeta X^{*}(1-\Lambda)\left(Z-X^{*}\right)}-1 \text {, }
$$

$$
\frac{X^{*}}{X}=\left\{(1-\Lambda)^{-2}+\frac{\Omega^{4} d^{2}}{g^{2} \chi^{2}}\right\}^{1 / 2}\left\{1+\frac{\Omega^{4} d^{2}}{g^{2} \chi^{2}}\right\}^{-1 / 2} .
$$

We cannot, of course, solve $\left(13^{\prime}\right)$ for $\zeta$; but it can be shown that since $X^{*}>X$, equation (13) gives values of $\zeta$ which are too large, that is, the actual values of $\zeta$ should be less than those shown in figure 3 . It follows that the values of $a / b$ should be larger than those shown in figure 4 .

We can obtain a solution of $\left(12^{\prime}\right)$ and $\left(13^{\prime}\right)$ if we suppose that $\Lambda$ is nearly equal to unity. This supposition is an extra relationship between the variables that might never be satisfied in practice, i.e. it might be inconsistent with equations derived from a study of other regions of the flow; consequently results obtained by its use may be inaccurate, but they should give an idea of the effect of not neglecting $\Lambda$. When $\Lambda \doteqdot 1$,

$$
\Omega^{4} d^{2} /\left(g^{2} \chi^{2}\right) \doteqdot \Omega^{2} k^{2} Z^{2} / W^{2} \ll 1,
$$

and so $X^{*} \doteqdot X /(1-\Lambda)$. From $\left(13^{\prime}\right)$, it follows that $X^{*}$ must be less than $Z$ if $r$ is to be real, and so $X$ must be small when $(1-\Lambda)$ is small. Thus, it is a good approximation to take $\zeta$ as the root of $X=0$, i.e. $\zeta=0 \cdot 3$. On combining equations (11), $\left(12^{\prime}\right)$ and $\left(13^{\prime}\right)$ with the condition that $\Lambda$ is nearly equal to unity, we obtain $X^{*} \div 2 Y Z(1+2 Y)^{-1} \div 0.65, r=1.8 \mathrm{~mm}$ and $W=25 \mathrm{~cm} / \mathrm{sec}$.

It can be shown further that $\Lambda$ cannot be larger than unity, and so we have the result that the spiralling motion ceases when $r=1.8 \mathrm{~mm}$, $W=25 \mathrm{~cm} / \mathrm{sec}, a / b=3.5$ and $\Omega d /\left(W_{\chi}\right)=0.54$. The velocity of rise is in closer agreement with the experimental results than the value obtained previously, but $a / b$ is too large and the maximum radius for spiralling bubbles is too small; thus it seems likely that the supposition of $\Lambda$ being nearly equal to unity is inconsistent. However, the above result indicates that, if $\Lambda$ were not neglected, the effect would be to give a maximum radius smaller than that obtained previously, a slightly increased $W$ and an increased $\Omega d /\left(W_{\chi}\right)$ for bubbles whose radius is nearly equal to the maximum radius.

Although, as we have seen, the neglect of $\Lambda$ may account for some of the discrepancies between theory and experiment, it will not account for the discrepancies between the calculated and observed velocities of rise, especially when the bubbles are small. A possible explanation lies in the assumption that the bubble is exactly an oblate spheroid. If the bubble were spherical, of radius $R$, equation (11) would become $W=\frac{2}{3}(g R)^{1 / 2}$; and when, for instance, $R=1.0 \mathrm{~mm}, W$ would be $6.7 \mathrm{~cm} / \mathrm{sec}$. It is therefore clear that the calculated velocity of rise is sensitive to the assumed 
shape of the bubble. Now the surface tension enters into the above equations as a product with the rate of variation along the surface of the sum of the principal curvatures, and if this rate were larger, then so would be the velocity of rise. There are thus reasons for expecting that if the assumed shape of the bubble had greater variations of curvature than an oblate spheroid, as may well be the case, then better agreement with experiment would be obtained. But if such a shape were assumed, the mathematical analysis would become much more difficult, if, indeed, possible at all.

\section{Separation of the flow}

The analysis can be modified in order to take some account of the possibility that the flow around the bubble separates. It was previously assumed that the flow at the front of the bubble is the same as the ideal flow around a body of the same shape. However, there is evidence of a wake behind a spiralling bubble (see, for example, Miyagi 1925), and this implies that the flow around the bubble separates. It is still reasonable to suppose that the flow near the front is irrotational, but the flow will be different from that given by expression (3). The principal difference will probably be in the gradient of the velocity at the stagnation point, and it is unlikely that equations (4) and (5), which determine the position of the front stagnation point, are seriously in error. Equations (7) and (8) will still be correct, since they arise from the first derivatives in (6) evaluated at the stagnation point, and are independent of the velocity.

However, equation (11) needs modifying since it involves $\partial^{2} \mathbf{V}^{2} / \partial s^{2}$ (where $\partial / \partial s$ denotes differentiation along the surface), and this will affect the coefficient of $W^{2}$. It should be replaced by

$$
\frac{W^{2}}{g k}=\frac{K Z^{2}}{1+\zeta^{2}}\left(\frac{4 \zeta T}{\rho g k^{2}\left(1+\zeta^{2}\right)^{2}}+\zeta\right)
$$

where $K$ is the value of $\partial^{2} \mathbf{V}^{2} / \partial s^{2}$ for the ideal flow divided by the value for the separation flow. Typical values of $K$ are probably obtained by comparing the ideal flow around a sphere with the ideal flow around a variety of Rankine solids, it being supposed that the separation flow around the sphere corresponds to the ideal flow around the solid. Values of $K$ between one and two are then obtained, depending upon the shape of the solid; and it seems plausible to suppose that the values of $K$ for the flow around a bubble will also lie in this range.

Equation (12) will then be replaced by

and equation (13) by

$$
\frac{\Omega d}{W \chi}=\frac{X}{K Z}
$$

$$
\frac{4 T}{\rho g k^{2}\left(1+\zeta^{2}\right)^{2}}=\frac{1+\zeta^{2}}{2 Y \zeta(Z-X / K)}-1
$$

If $K$ is less than $1 \cdot 5$, the solutions are similar to those for $K=1$, which have already been given. The limit $r=0$ still corresponds to a finite value of $\zeta$, although this value is larger than 1.52 and tends to infinity as 
$K$ tends to $1 \cdot 5$. Also, $W$ tends to infinity as $r$ tends to zero, but is less or greater than the corresponding values for $K=1$, according as $r$ is less or greater than some value in the neighbourhood of $1.4 \mathrm{~mm}$ (the exact value of $r$ at the 'cross-over' depending on $K$ ), but the values of $W$ are still close to those for $K=1$.

However, if $K$ is greater than $1 \cdot 5, \zeta$ tends to infinity as $r$ tends to zero, while $W$ is zero when $r=0$ and increases monotonically with $r$, crossing the curve corresponding to $K=1$ in the neighbourhood of $1.6 \mathrm{~mm}$. In all cases, however, there is a maximum value for the radius of a spiralling bubble which is close to the maximum value that was obtained with $K=1$. The solutions of equations $\left(11^{\prime}\right),\left(12^{\prime \prime}\right)$ and $\left(13^{\prime \prime}\right)$, with a typical value $K=1 \cdot 75$, are shown in figures 3 and 5 .

Of special interest is the result that the curves of $W$ against $r$, when $K$ is greater than 1.5 , are similar in shape to the experimental curve for air bubbles in tap water or water containing surface-active agents obtained by Haberman \& Morton (see figure 5), the theoretical values of $W$ with $K=1.75$ being about $10 \%$ too small. (Closer agreement could be obtained here by choosing $K$ smaller than 1.75 , but this is of little significance.) Thus, the theory can be made consistent with the experimental observations of bubbles in tap water, provided that we postulate increased separation of the flow and the possibility that $K$ may be greater than $1 \cdot 5$.

It has been suggested in the literature that the effect of impurities or surface-active substances is to make the surface of the bubble more 'solid', thus increasing the drag and reducing the velocity of rise. This suggestion is consistent with the above result, since if the surface of the bubble is more 'solid', then, presumably, separation will occur earlier and $K$ will be increased. The fact that the drag in greater also suggests that separation occurs earlier, since the skin friction is probably small compared with the form drag (at a pure air-water interface, the skin friction is zero), and an earlier separation of the flow usually accompanies a marked increase in form drag. The increase in drag has been associated with the existence of circulation of the air inside the bubble, but it is difficult to see how a motion of the air can appreciably affect the motion of the water, since the density and viscosity of air are so small compared with those of water.

It is to be emphasized, however, that there is no direct evidence which relates the presence of impurities to the separation and thence to the value of $K$; and until such evidence is forthcoming, these ideas are to be treated with reserve.

It is perhaps worth mentioning here that Davies \& Taylor (1950) found that the flow near the nose of a large spherical-cap bubble was close to the ideal flow past the sphere of which the bubble was the cap. Since photographs of these bubbles indicated that a bubble and its wake together had approximately the shape of a sphere, so that the dividing streamlines lay approximately on the surface of a sphere, this result is not in contradiction with the above concept of separation of the flow. 
The velocities of spiralling bubbles in liquids other than water have been measured by Haberman \& Morton (1953), and the curves of velocity of rise against radius are similar in shape to the experimental curve $(a)$ shown in figure 5, except that the peaks are not quite so sharp. It follows from equations (11) and (13) that the dependence of $W(T / \rho)^{-1 / 4}$ on $r(T / \rho)^{-1 / 2}$ is the same for all liquids, and the data given by Haberman \& Morton satisfy this criterion with reasonable accuracy.

\section{The STABILITy of A RISING BUBble}

The method of $\S 3$ will be applied in this section to the motion of a zig-zagging spheroidal bubble. (As mentioned previously, we shall obtain from the analysis an equation which determines the stability of the rectilinear motion of a spheroidal bubble.) It is necessary to take into account the effects of variations with time of the shape, orientation and velocity of the bubble, since the motion of a zig-zagging bubble is not steady. To make the analysis tractable, such variations will be treated as small perturbations of the rectilinear motion. However, to account simultaneously for variations of these three quantities would still make the analysis unduly complicated; and, in order to find whether any simplifying assumptions are permissible, we shall first treat the case in which the unperturbed shape is taken to be a sphere instead of a spheroid. The results for a spherical bubble will enable us, in fact, to make plausible assumptions about the motion of a spheroidal bubble and to simplify the analysis considerably.

\section{The motion of a spherical bubble}

We calculate the velocity and pressure of the water at the front of the bubble using the same assumptions as in $\S 3$, that is, supposing the flow to be the ideal flow around a body of the same shape as the bubble. The unperturbed shape is taken to be the sphere $r=R$, and the perturbed shape to be $r=R\left(1+\epsilon_{0} S_{0}+\epsilon_{1} S_{1}+\epsilon_{2} S_{2}\right)$, where $\epsilon_{0} S_{0}=\epsilon_{0}(t), \epsilon_{1} S_{1}=\theta x+\phi y+\psi z$, and $\epsilon_{2} S_{2}=\lambda x^{2}+\mu y^{2}+\nu z^{2}+2 \alpha y z+2 \beta z x+2 \gamma x y$, where $\lambda+\mu+\nu=0$. It is assumed that sufficient generality is obtained by taking the perturbed shape as an ellipsoid and neglecting higher harmonics. The unperturbed velocity of rise is denoted by $\mathbf{W}=(W, 0,0)$, where the $x$-axis is taken vertically upwards. Perturbations of the velocity of the centre of the bubble can easily be shown to be equivalent to the term $\epsilon_{1} S_{1}$, that is, a perturbation $(u, v, w)$ of the velocity may be represented by $\epsilon_{1} S_{1}$ with $R^{2}(\dot{\theta}, \dot{\phi}, \dot{\psi})=(u, v, w)$.

We denote the velocity potential by $\Phi$. Then $\Phi$ satisfies $\nabla^{2} \Phi=0$, and $\Phi \rightarrow 0$ as $r \rightarrow \infty$. At the surface of the bubble, $\partial \Phi / \partial n=\partial r / \partial t+\mathbf{W}$. $\mathbf{n}$, where $\mathbf{n}$ denotes the unit normal to the surface. It follows, after some algebra, that

$$
\begin{aligned}
\Phi & =-\frac{W x R^{3}}{2 r^{3}}+\sum_{n=0}^{2} \epsilon_{n}\left\{-\frac{R^{2} \dot{S}_{n}}{n+1}\left(\frac{R}{r}\right)^{2 n+1}-\right. \\
& \left.-\frac{3 W}{2}\left(\frac{R}{r}\right)^{2 n+3}\left(x S_{n}-\frac{r^{2}}{2 n+1} \frac{\partial S_{n}}{\partial x}\right)+\frac{3}{2} \frac{W R^{2}(n-1)}{n(2 n+1)}\left(\frac{R}{r}\right)^{2 n-1} \frac{\partial S_{n}}{\partial x}\right\},
\end{aligned}
$$


where the dot denotes differentiation with respect to time, and the term with $n$ in the denominator is to be omitted when $n=0$. The pressure is given by

$$
\frac{p}{\rho}+\frac{1}{2}(\nabla \Phi)^{2}+d \Phi / d t+g x=\text { constant, }
$$

where $d / d t \equiv(\partial / \partial t-W \partial / \partial x)$. Also, the sum of the principal curvatures of the surface is (see Lamb 1932, §275)

$$
\frac{1}{R_{1}}+\frac{1}{R_{2}}=\frac{1}{2 R}+\sum_{n=0}^{2}(n-1)(n+2) \epsilon_{n} S_{n} / R
$$

Hence, the value of $p+T\left(1 / R_{1}+1 / R_{2}\right)$ at the surface of the bubble is known. As in $\S 3$, we suppose that $p+T\left(1 / R_{1}+1 / R_{2}\right)$, when calculated in this manner, is such that its first and second derivatives (differentiating along the surface) vanish at the point $y=z=0$.

After some algebra, we obtain the following equations:

$$
\ddot{\alpha}+\frac{15}{2} \frac{W \dot{\alpha}}{R}+\left(\frac{12 T}{\rho R^{3}}+\frac{3 g}{R}\right) \alpha=0
$$

and the same equation with $\alpha$ replaced by $\mu-\nu$; also

$$
\ddot{\gamma}+\frac{93 W \dot{\gamma}}{20 R}+\gamma\left(\frac{12 T}{\rho R^{3}}-\frac{21 g}{10 R}\right)+\frac{3 \dot{v}}{4 R^{3}}+\frac{27}{8} \frac{W v}{R^{4}}=0
$$

and the same equation with $\gamma$ and $v$ replaced by $\beta$ and $w$ respectively; and

$$
\begin{aligned}
\frac{9 W^{2}}{4 R^{2}}-\frac{g}{R}+\frac{3}{2} \frac{W \dot{\epsilon}_{0}}{R}-\frac{g \epsilon_{0}}{R}+ & \frac{1}{2} \frac{\dot{u}}{R}+\frac{9}{4} \frac{W u}{R^{2}}+ \\
& +R^{2} \ddot{\varkappa}+\frac{81}{10} W R \dot{\lambda}+\lambda\left(\frac{12 T}{\rho R}-\frac{8 g R}{5}\right)=0 .
\end{aligned}
$$

If the first and second derivatives of $p+T\left(1 / R_{1}+1 / R_{2}\right)$ evaluated at some other point of the surface had been put equal to zero, then the same equations would have been obtained provided the distance between this point and $y=z=0$ was of the order of $\epsilon$. For extra terms of this order would arise only if the third derivatives of $p+T\left(1 / R_{1}+1 / R_{2}\right)$ evaluated at $y=z=0$ were not small, whereas, since

$$
p+T\left(\frac{1}{R_{1}}+\frac{1}{R_{2}}\right)=-\rho g x-\frac{9}{8} \rho \frac{W^{2}}{R^{2}}\left(y^{2}+z^{2}\right)+0(\epsilon)
$$

the third derivatives are in fact of the order of $\epsilon$.

It follows from equation (14) that the bubble shape is always stable to perturbations of $\alpha$ and $\mu-\nu$, and that such perturbations are heavily damped. It can be shown that the deviation from axial symmetry about the vertical of the shape of the bubble is dependent upon these quantities. Equations (15) and (16) involve more than one variable, and no informtaion about stability can be obtained without the aid of other relations between the variables; how such relations are to be obtained is not clear. Of importance, however, is the result that $\gamma$ and $v$ occur together in an equation 
which does not involve any of the other variables. The meaning of $\gamma$ is clear when the perturbation is symmetrical about the plane $x, y$, and $\epsilon_{2} S_{2}$ can be taken as $\lambda x^{2}+\mu y^{2}+\nu z^{2}+2 \gamma x y$. Then $\gamma$ is proportional to the angle between the principal axes of the distorted sphere and the coordinate axes, so that equation (15) involves only the sideways velocity $v$ and the orientation of the bubble.

\section{The zig-zagging spheroidal bubble}

As pointed out at the beginning of $\S 3$, the assumption that a zig-zagging bubble is spherical is a bad one. For example, equation (16) predicts that $W \doteqdot \frac{2}{3}(g R)^{1 / 2}$, which gives values of $W$ that are too small by a factor of three. We shall therefore assume that the bubble is an oblate spheroid. Further, we shall neglect changes of shape and vertical velocity, and consider variations of the sideways velocity and the orientation of the bubble only. There are two reasons for this. Firstly, it seems plausible to assume, by analogy with the equations obtained above for a spherical bubble, that departures from axial symmetry are heavily damped, and that the equation involving the sideways velocity and orientation of the bubble is independent of perturbations of the vertical velocity and shape. Secondly, it is variations of the sideways velocity and orientation only that are directly observed.

We take axes $(x, y, z)$ fixed in the bubble, $x$ being the axis of symmetry, and $x, y$ the vertical plane containing the zig-zag. Let $\chi$ (measured in the positive sense from $x$ ) denote the angle between the $x$-axis and the vertical. We suppose that the bubble moves with constant vertical velocity $W$ and variable horizontal velocity $v$ in the plane $x, y$. It is supposed that $\chi$ and $v$ are small, and their squares and products are neglected.

Referred to the axes $(x, y, z)$ the bubble has velocity $(W, W \chi+v, 0)$ and angular velocity $(0,0,-\dot{\chi})$. We use oblate spheroidal coordinates $\zeta$, $\mu$ and $\omega$ (see $\S 3$ ). Then the velocity potential $\Phi$ is given by

$$
\begin{aligned}
& \Phi= k W\left\{\zeta_{0}\left(1+\zeta_{0}^{2}\right)^{-1}-\cot ^{-1} \zeta_{0}\right\}^{-1} \mu\left(1-\zeta \cot ^{-1} \zeta\right)+k(v+W \chi) \times \\
& \times\left\{\frac{\zeta_{0}^{2}+2}{\zeta_{0}\left(\zeta_{0}^{2}+1\right)}-\cot ^{-1} \zeta_{0}\right\}^{-1}\left(1-\mu^{2}\right)^{1 / 2}\left(1+\zeta^{2}\right)^{1 / 2}\left\{\frac{\zeta}{\zeta^{2}+1}-\cot ^{-1} \zeta\right\} \cos \omega+ \\
&+A \mu\left(1-\mu^{2}\right)^{1 / 2}\left(1+\zeta^{2}\right)^{1 / 2}\left\{3 \zeta \cot ^{-1} \zeta-3+\left(1+\zeta^{2}\right)^{-1}\right\} \cos \omega,
\end{aligned}
$$

where the surface of the bubble corresponds to $\zeta=\zeta_{0}$, and

$$
A\left[\frac{\partial}{\partial \zeta}\left\{\left(\zeta^{2}+1\right)^{1 / 2}\left[3 \zeta \cot ^{-1} \zeta-3+\left(1+\zeta^{2}\right)^{-1}\right]\right\}\right]_{\zeta=\zeta_{0}}=k^{2} \dot{\chi}\left(1+\zeta_{0}^{2}\right)^{-1 / 2}
$$

The pressure is given by

$$
p / \rho+\frac{1}{2}(\nabla \Phi)^{2}-\mathbf{U} \cdot \nabla \Phi+\partial \Phi / \partial t+g H=\text { const., }
$$

where $\mathbf{U}=(W, W \chi+v, 0)+(\dot{\chi} y,-\dot{\chi} x, 0)$ and $H=x \cos \chi+y \sin \chi$.

To determine $v, \chi$ and $W$, we shall use the condition that the first and second derivatives along the surface of $p+T\left(1 / R_{1}+1 / R_{2}\right)$ vanish at the 
point where the dividing streamline meets the surface, ${ }^{*}$ this point being given by $\nabla \Phi=\mathbf{U}$.

After some algebra, it is found that the coordinates of this point are given by

$$
\omega=0, \quad \sin \theta=\frac{Z}{W\left(1+\zeta^{2}\right)^{1 / 2}}(2 Y(v+W \chi)-X k \dot{\chi}),
$$

where $\mu=\cos \theta$, and the suffix of $\zeta_{0}$ has been dropped since all expressions are evaluated on $\zeta=\zeta_{0}$. We put $\mathbf{q}=\nabla \Phi-\mathbf{U}$, and rewrite equation (17) as

$$
\frac{p}{\rho}+\frac{1}{2} \mathbf{q}^{2}-\frac{1}{2} \mathbf{U}^{2}+\frac{\partial \Phi}{\partial t}+g H=\text { const. }
$$

On neglecting squares and products of $\chi$ and $v$, we obtain

$$
\begin{aligned}
& \mathbf{q}^{2}=\frac{W^{2}\left(1-\mu^{2}\right)\left(1+\zeta^{2}\right)^{2}}{\left(\mu^{2}+\zeta^{2}\right) Z^{2}}-\frac{2 W\left(1+\zeta^{2}\right)^{3 / 2}\left(1-\mu^{2}\right)^{1 / 2}}{\left(\mu^{2}+\zeta^{2}\right) Z} \cos \omega \times \\
& \times\left(2(v+W \chi) Y \mu+\dot{\chi} k\left[(X-\zeta)\left(1-2 \mu^{2}\right)-\zeta\right]\right), \\
& \mathbf{U}^{2}=W^{2}+2 W k \dot{\chi}\left(1+\zeta^{2}\right)^{1 / 2} \sin \theta \cos \omega, \\
& \frac{\partial \Phi}{\partial t}=-k(\dot{v}+W \dot{\chi}) \zeta Y Z\left(1+\zeta^{2}\right)^{-1 / 2} \cos \omega \sin \theta+ \\
& +k^{2} \ddot{\chi}\left(1+\zeta^{2}\right)^{1 / 2}(X-\zeta) \cos \theta \sin \theta \cos \omega .
\end{aligned}
$$

and

Further,

$$
\frac{1}{R_{1}}+\frac{1}{R_{2}}=\frac{\zeta\left(1+\zeta^{2}\right)^{1 / 2}}{k}\left\{\frac{1}{\left(\zeta^{2}+\mu^{2}\right)^{3 / 2}}+\frac{1}{\left(\zeta^{2}+1\right)\left(\zeta^{2}+\mu^{2}\right)^{1 / 2}}\right\}
$$

We now apply the boundary condition on the pressure. The first derivative with respect to $\omega$ vanishes identically. The vanishing of the first derivative with respect to $\theta$ gives, after some algebra,

$$
\begin{aligned}
\left(\frac{4 T \zeta}{k^{2}\left(\zeta^{2}+1\right)^{2}}+g \zeta\right)\left(1+\zeta^{2}\right)^{-1 / 2} & \sin \theta \\
& =-W \dot{\chi}-(\dot{v}+W \dot{\chi}) \frac{\zeta Y Z}{1+\zeta^{2}}+g \chi+k \ddot{\chi}(X-\zeta) .
\end{aligned}
$$

The second derivative with respect to $\theta$ and $\omega$ vanishes identically. The second derivative with respect to $\theta$ gives

$$
\frac{W^{2}}{g k}=\frac{Z^{2}}{1+\zeta^{2}}\left(\frac{4 T \zeta}{\rho g k^{2}\left(1+\zeta^{2}\right)^{2}}+\zeta\right)
$$

and the second derivative with respect to $\omega$ is a combination of equations (18), (19) and (11).

On eliminating $\theta$ between equations (18) and (19), we obtain

$$
k(\zeta \chi-X)+W \dot{\chi}\left\{1+\frac{\zeta Y Z}{1+\zeta^{2}}-\frac{X}{Z}\right\}+\chi\left\{\frac{2 Y W^{2}}{k Z}-g\right\}+\frac{\dot{v} \zeta Y Z}{1+\zeta^{2}}+\frac{2 W Y v}{k Z}=0 .
$$

* It is really only a matter of convenience which point is taken for the vanishing of the derivatives, since it can easily be shown that, provided the distance between the point chosen and the axis of symmetry is of order $\chi$, the same equations will result from the analysis. 
If $\zeta \rightarrow \infty$ and $k \rightarrow 0$ in such a way that $k \zeta \rightarrow R$ (this is the limiting process by which the spheroid becomes a sphere), it can be shown that equation (20) reduces to equation (15).

Now equation (20) is an equation in the two variables $v$ and $\chi$, and it is not possible to proceed further without another relation between these variables. It is by no means clear how such a relation is to be obtained, although, presumably, one could be obtained from a consideration of the forces and couples on the bubble. However, these forces and couples cannot be calculated accurately without a knowledge of the motion in the wake. The simplest hypothesis that we can make in order to find another relation between $v$ and $\chi$ is to suppose that the actual couple on the bubble is proportional to the couple on a body of the same shape as the bubble moving with the same velocity in an ideal liquid. This couple is easily calculated (see, for example, Lamb 1932, §124). Now, the total couple on the bubble is zero, since the mass of the air inside the bubble is negligible, and we hence obtain the result

$$
\begin{gathered}
R k^{2} \ddot{\chi}+W(v+W \chi)=0, \\
\text { where } R=\frac{1}{5} \zeta Z\left(1+\zeta^{2}\right)^{-1}\left\{2 Y\left(3+4 \zeta^{2}\right)-3\left(1+2 \zeta^{2}\right)\right\}^{-1}
\end{gathered}
$$

Now, the slowest natural frequency of oscillation of a stationary air bubble of radius $R$ in water is $(1 / 2 \pi)\left(12 T / \rho R^{3}\right)^{1 / 2}$. When $R$ is about one millimetre, this frequency is about 150 per second; and, further, equations (14), (15) and (16) indicate that when air bubbles of about this size are rising freely, this natural oscillation is not sensibly affected and remains stable. The frequency of the zig-zag motion is about seven per second, which is much slower, and we shall confine ourselves here to perturbations which have a time scale comparable with the time scale $\tau=1 / 7$ second, say, of the zig-zag motion. That is, we shall neglect the natural oscillations of the bubble because they are too rapid and are apparently stable. Then

$$
R k^{2} \ddot{\chi} /\left(W^{2} \chi\right)=O\left(k^{2} /\left(W^{2} \tau^{2}\right)\right)=O\left(10^{-3}\right)
$$

(this equation represents the fact that the velocity perturbations due to the oscillation of the bubble are small compared with the velocity of rise), and (21) reduces to

$$
v+W \chi=0
$$

which is the condition that the velocity of the bubble is parallel to the axis of symmetry. This result is similar to the assumption made in $\S 3$ that the axis of symmetry and the velocity of a spiralling bubble are nearly parallel; but, in the case of a zig-zagging bubble, there is no experimental evidence either to contradict or confirm (22), since the photographs of zig-zagging bubbles that have so far been taken are not good enough for this purpose. We shall see, however, that the use of (22) leads to results in reasonable agreement with experiment.

On substituting (22) in equation (20) and neglecting terms which are of order $k /\left(W_{\tau}\right)=O(1 / 30)$, we obtain

$$
W \dot{\chi}(Z-X)-g Z \chi=0 .
$$


Equation (23), which is the final result of our analysis of the zig-zag motion, is essentially a stability equation and its implications will be discussed in the next sub-section.

\section{The stability of a rising bubble}

Equation (23) implies that $\chi$ is damped exponentially when $\zeta>1.52$, and increases exponentially when $\zeta<1 \cdot 52$, since $Z-X$ is a monotonically decreasing function of $\zeta$ which is zero when $\zeta=1 \cdot 52$. For $\zeta$ nearly equal to $1 \cdot 52$, equation (23) gives a large value to $\dot{\chi} / \chi$ and is inconsistent with the approximations by which it is derived. Hence, for $\zeta$ nearly equal to 1.52 , equation (22) does not hold, or it is not valid to neglect changes of shape and vertical velocity, or, as seems most likely, a linearized theory is not valid and second-order terms should be taken into account. The exponential increase of $\chi$ when $\zeta<1.52$ also implies that a linearized theory is not valid for long in this case.

Thus, the analysis of the motion near the front of the bubble does not give us any information about the zig-zag motion, but gives the result that if the bubble is more oblate than the spheroid corresponding to $\zeta=1.52$, then it is unstable to small disturbances and will move in an irregular manner, although the above equations do not indicate in what way it will move. (It will be remembered that in $\S 3$ we found $\zeta<1.52$ to be a necessary condition for the spiralling motion to occur.) In other words, we have found the value of $\zeta$ for which the rectilinear motion of a spheroidal bubble becomes unstable. It is true that we have considered, in effect, only disturbances of sideways velocity and orientation which are symmetrical about a plane, but this is probably sufficient, both for the reasons given before in the sub-section on the zig-zagging spheroidal bubble and because the experimental observations of $\S 2$ show that the effect of the first instability is to produce a zig-zag motion in a plane. The possible influence of the motion in the wake on the occurrence of the instability and the mechanism of the zig-zag motion will be discussed later.

Let us now compare the above prediction with the experimental data for the critical bubble, i.e. the smallest bubble that is unstable. From equation (11) with $\zeta=1 \cdot 52, T=74$ dynes $/ \mathrm{cm}$ and $g=981 \mathrm{~cm} / \mathrm{sec}^{2}$, we obtain, as the relation between the velocity and radius of the critical bubble,

$$
W^{2}=223 k^{-1}+81 k,
$$

where $r=1.7 k$ (using $r=k\left\{\zeta\left(1+\zeta^{2}\right)\right\}^{1 / 3}$ ), $k$ is measured in millimetres and $W$ in $\mathrm{cm} / \mathrm{sec}$. The observed values are $r=0.7 \mathrm{~mm}$ and $W=30 \mathrm{~cm} / \mathrm{sec}$ approximately. On putting $r=0.7 \mathrm{~mm}$ in (24), we obtain $W=24 \mathrm{~cm} / \mathrm{sec}$, $(W=30 \mathrm{~cm} / \mathrm{sec}$ corresponds to $r=0.4 \mathrm{~mm})$. This velocity is somewhat lower than the experimental value, as was also the case in $\S 3$ for the calculated velocity of a spiralling bubble, and the reasons that were put forward there to account for the disagreement will apply here with equal force. It is also worth mentioning that when $\zeta=1 \cdot 52$, the ratio of the major to the minor axis of the bubble is $1 \cdot 2$, which agrees well with the 
experimental value for a bubble of radius $0.7 \mathrm{~mm}$ given by Rosenberg (1950) and shown in figure 4.

Further, on neglecting $\rho g k^{2}\left(1+\zeta^{2}\right)^{2} /(4 T)$, which is usually $O\left(10^{-1}\right)$ for the critical bubble (this is equivalent to neglecting the second term on the right-hand side of (24)), equation (11) gives the value of the critical Weber number $2 W^{2} r \rho / T$ at which the instability appears. We have

$$
\left(\frac{2 W^{2} r \rho}{T}\right)_{\text {crit. }}=\left\{\frac{8 Z^{2} \zeta^{4 / 3}}{\left(1+\zeta^{2}\right)^{8 / 3}}\right\}_{\zeta=1.52}=1.03,
$$

whereas the experimental value for air bubbles in water is about 1.7.

For the sake of completeness, we shall also investigate briefly the effect of separation of the flow on the relation between the velocity and radius of the critical bubble. As before, only equation (11) requires modification, and on replacing it by $\left(11^{\prime}\right)$ and repeating the analysis, we obtain

$$
W \dot{\chi}(Z-X \mid K)-g Z \chi=0 .
$$

The motion becomes unstable when $\zeta$ is equal to the root of $Z-X / K=0$, and from (11'), we obtain the required relation between the velocity and radius of the critical bubble. It is found that for a given radius, the velocity decreases as $K$ increases. For example, $W=14 \mathrm{~cm} / \mathrm{sec}$ corresponds to $r=0.7 \mathrm{~mm}$ when $K=4 / 3$. These figures are consistent with the observations of $\S 2$ that bubbles in tap water start to zig-zag when their radii are about $0.8 \mathrm{~mm}$, even though their velocities of rise are much smaller than bubbles of the same size in filtered or distilled water. However, if $K>3 / 2, Z-X / K$ is always positive, and this implies that if the motion is such that $K>3 / 2$, then it is unstable for all $\zeta$.

\section{The mechanism of the zig-zag motion}

We have not so far considered the motion in the wake of the bubble, since no way could be found of overcoming the mathematical difficulties. However, there is some experimental evidence on the wake, which we shall now describe, and it will be seen that it suggests that the zig-zag motion and the structure of the wake are in some way related.

Figure 6 shows the dependence of the drag coefficient $C_{D}=(8 / 3)\left(g r / W^{2}\right)$ upon the Reynolds number $2 W r / \nu$. This curve was constructed from data on the velocity of rise of zig-zagging bubbles given by Datta, Napier \& Newitt (1950). (Haberman \& Morton (1953) give a curve for spiralling bubbles which is very similar.) It will be noticed that the transition to zig-zag motion is accompanied by a rapid increase in the drag coefficient. All the drag is form drag, there being no skin friction since the boundary condition at an air-water interface is zero tangential stress instead of zero velocity of slip, and an alteration in the behaviour of the drag coefficient must be associated with a marked change in the structure of the wake. There is no direct evidence on the wake behind bubbles, but there is some on the wake behind solid three-dimensional bodies, and this we shall now describe briefly. 
Marshall \& Stanton (1930) observed the flow past a circular disc which was normal to the direction of flow, and found that the wake was steady if the Reynolds number was less than 200. When the Reynolds number became equal to 200 , a periodic discharge of vorticity, symmetrical about a plane perpendicular to the disc, was observed. Moeller (1938) observed the wake behind a solid sphere over a large range of Reynolds numbers. He found that for Reynolds numbers less than 170 , the wake was steady and laminar, but for Reynolds numbers greater than 200 , the wake was no longer steady but oscillated periodically. The amplitude of the oscillation increased with the Reynolds number, being initially quite small, until the wake became turbulent at a Reynolds number of about 1000 , the turbulent wake still showing signs of a periodic structure. The oscillation of the laminar wake was symmetrical about a plane and there was no evidence at all of a spiral wake.

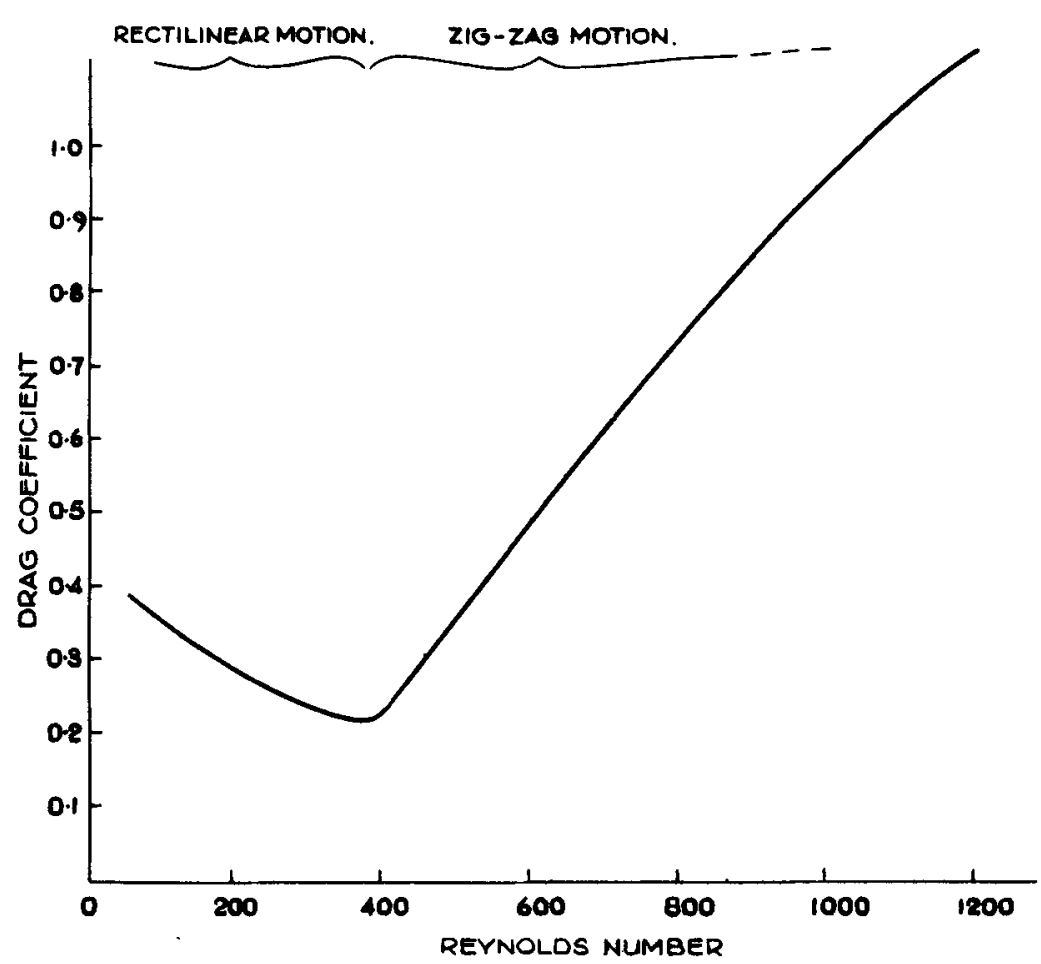

Figure 6. Dependence of drag coefficient on Reynolds number for zig-zag motion.

Moeller gives the frequency of oscillation of the wake $N$ for Reynolds numbers larger than 800 ; for instance, when $2 W r / \nu=800, N r / W=0 \cdot 12$. Marshall \& Stanton give $N r / W=0.06$ for a Reynolds number of 200 only.

The value of $\mathrm{Nr} / \mathrm{W}$ for a zig-zagging bubble is about 0.02 at Reynolds numbers which are greater than 400 , this being the Reynolds number of 
the critical bubble. However, we should expect the wake behind a bubble to be somewhat 'weaker' than the wake behind a solid body, owing to the different boundary conditions and consequent reduction in the rate of vorticity production; and it seems likely that an oscillation, or instability, of the wake of a bubble would first appear at a higher Reynolds number and have a smaller frequency than the oscillations of the wake of a solid body. It is therefore possible that the zig-zag motion is related to an oscillation of the wake or a periodic discharge of vorticity from behind the bubble, in so far as these may be distinguished in the light of present knowledge.

We therefore suggest that the observed zig-zag motion is caused by the interaction of an oscillating wake and the instability of the motion near the front of the bubble that occurs when the bubble is sufficiently oblate (i.e. when $\zeta$ is less than 1.52). It is unlikely that the wake oscillation by itself could produce the zig-zag, since similar motion has not apparently been observed with solid bodies. A familiar phenomenon that might seem to be relevant is the motion of a falling leaf; however, this has a different character, since the leaf glides through the air with its velocity in the plane of the leaf, whereas a bubble moves with its velocity perpendicular to its equatorial plane. Also, the argument that the forces arising from the wake oscillation are not sufficient to move a solid body sideways but would be sufficient to move a bubble is not valid, since a bubble has, in fact, a virtual mass, this being the mass of the water that has to be accelerated when the bubble accelerates. It may be that the instability at the front of the bubble occurs first and 'triggers' off an oscillation of the wake. The marked change in the behaviour of the drag coefficient makes this seem likely, but the actual details of how the zig-zag motion is produced are not clear.

This suggested mechanism also fits the data and analysis for bubbles in tap water, unless $K$ is greater than $\frac{3}{2}$ and the front of the bubble is unstable for all $\zeta$. In this case, we should expect the motion to be irregular until the Reynolds number is high enough for the oscillation of the wake to be possible. (It will be remembered that the analysis is only valid when the Reynolds number is sufficiently large for the assumption of inviscid flow near the front of the bubble to hold.) There is no evidence of this happening, and $K$ is presumably less than $\frac{3}{2}$. This does not necessarily contradict the suppcsition in $\S 3$ that $K$ for spiralling bubbles may be greater than $\frac{3}{2}$, since $K$ will depend on the Reynolds number and the nature of the motion.

Finally, there is no evidence concerning the causes of the spiralling motion, except that the experimental observations make one wonder whether they have anything to do with the onset of turbulerse in the wake.

The author wishes to thank Dr G. K. Batchelor for suggesting this problem to him and for much helpful advice and encouragement, and the Department of Scientific and Industrial Research for a maintenance award. 


\section{REFERENCES}

Datta, R. L., Napier, D. H. \& Newitt, D. M. 1950 Trans. Instn. Chem. Engrs. $28,14$.

Davies, R. M. \& Taylor, G. I. 1950 Proc. Roy. Soc. A, 200, 375.

Haberman, W. L. \& Morton, R. K. 1953 David Taylor Model Basin, Rep. no. 802.

LAmB, H. 1932 Hydrodynamics, 6th Ed. Cambridge University Press.

Marshall, D. \& Stanton, T. E. 1930 Proc. Roy. Soc. A, 130, 295.

Miyagi, O. 1925 Phil. Mag. (6th Series), 50, 112.

Moelder, W. 1938 Phys. Zeit. 39, 57.

Peebles, F. N. \& Garber, H. J. 1953 Chem. Eng. Prog. 49, 88.

Rosenberg, B. 1950 David Taylor Model Basin, Rep. no. 727. 